\title{
DOCENCIA DE LA ASIGNATURA "ENERGÍA DE LA BIOMASA" EN EL MÁSTER EUROPEO EN ENERGÍAS RENOVABLES: LA EXPERIENCIA DEL GRUPO BERA
}

\author{
Maryori Díaz, Daniel García, \\ Maider Gómez, Eva López, \\ Daniel Maraver, Adeline Rezeau, \\ Fernando Sebastián \\ Centro de Investigación de Recursos y \\ Consumos Energéticos
}

\begin{abstract}
RESUMEN: Para afianzar y desarrollar las capacidades de los alumnos de posgrado de la asignatura denominada "Energía de la Biomasa" del Máster Europeo en Energías Renovables, el Grupo BERA (Biomasa, Evaluación, Recursos y Aprovechamiento) del Centro de Investigación CIRCE (Centro de Investigación de Recursos y Consumos Energéticos) lleva a cabo un conjunto de prácticas orientadas a motivar y educar al alumno en la realización de trabajos y presentaciones grupales en un período de tiempo limitado. De esta manera el alumno debe afrontar situaciones similares a las que podrá encontrarse durante el desarrollo de proyectos en el área de las Energías Renovables en un entorno empresarial y/o investigador. Las diferentes sesiones docentes que expone este artículo cumplen con el objetivo prioritario de afianzar los conocimientos adquiridos en las clases teóricas, además de lograr un segundo objetivo no menos importante, el aunar distintas disciplinas de la ingeniería y la economía, lo que se considera muy conveniente dada la heterogeneidad de la procedencia educativa de los alumnos que cursan el Máster. Este documento presenta las bases de la preparación de las sesiones, los objetivos que se pretenden alcanzar en las mismas y las conclusiones a las que llegaron los docentes tras su puesta en práctica.
\end{abstract}

ABSTRACT: In order to reinforce and develop the skills of the postgraduate students in the so-called "Energy from Biomass" subject within the European Masters in Renewable Energies, the BERA Group (Biomass, Evaluation, Resources and Use) of the CIRCE Research Institute (Centre for the Research of Energy Resources and Consumptions) carries out a series of practical sessions oriented to motivate and orientate the student in the execution of group work and presentations in a limited period of time. In this manner, the students must 
face similar situations to the ones that they would confront during the carrying out of real projects in the field of Renewable Energies in a business and/or research environment. The different teaching sessions analyzed in the present paper are oriented to achieve a main objective which is to consolidate the acquired knowledge in the theoretical sessions, as well as attaining a not less important objective: joining different disciplines of Engineering and Economy, which is very advisable due to the diversity of the students in the Master. This paper presents the basis for the session preparation, their desired objectives and the conclusions obtained by the teaching staff after their implementation.

PALABRAS CLAVE: Innovación didáctica, sesiones prácticas, Máster Europeo en Energías Renovables, biomasa.

KEYWORDS: Didactic innovation, practical sessions, European Master in Renewable Energies, biomass.

Recibido: $14 / 07 / 2010$

Aceptado: 15/10/2010

\section{INTRODUCCIÓN}

El objetivo general del Máster Europeo en Energías Renovables, diseñado e impartido por el Centro de Investigación CIRCE de la Universidad de Zaragoza, es la formación de posgraduados para especializarlos en la evaluación de recursos, así como en el diseño, en el análisis de viabilidad técnica y económica, en la optimización y en la gestión de instalaciones de aprovechamiento de Energías Renovables. Todo ello, teniendo en cuenta tanto el contexto social y económico actual como los escenarios socioenergéticos futuros.

El Máster está especialmente dirigido a los titulados en las áreas técnicas o científicas relacionadas con el aprovechamiento energético que tengan intención de desarrollar una carrera profesional o investigadora en el campo de las fuentes renovables y de la producción sostenible de energía. Por ello, las titulaciones requeridas para que los estudiantes sean admitidos al Máster son las siguientes: ingenieros e ingenieros técnicos (industrial, química, informática, etc.) o licenciados en áreas de ciencias.

Los objetivos generales del Máster son ofrecer una formación adaptada a las necesidades actuales de recursos humanos en el sector energético, proporcionar a los profesionales del sector una especialización actualizada y ampliar sus posibilidades de intercambio de información y experiencias.

El total de 12 créditos ECTS de la Asignatura "Energía de la Biomasa", en la que se centra el presente artículo, se distribuye en un módulo introductorio, cuatro módulos principales (Biocombustibles Sólidos, Biocarburantes, Biomasa Residual Húmeda y Residuos Sólidos Urbanos) y un módulo conclusivo. La docencia de estos seis módulos se coordina desde el grupo BERA (Biomasa, Evaluación, Recursos y Aprovechamiento) del Centro de Investigación CIRCE e incorpora una importante contribución de ponentes del sector empresarial y de la I+D en renovables que permiten tanto mantener una docencia con contenidos actualizados como aportar una visión amplia del sector. 


\section{OBJETIVOS PERSEGUIDOS Y METODOLOGÍA EMPLEADA}

Existen varios aspectos a tener en cuenta para el desarrollo de la innovación en la docencia: los métodos docentes y la documentación, por un lado, y el intercambio de experiencias y el reajuste de las prácticas, por otro (Zabalza, 2003-2004). Estos factores motivan al Grupo BERA a definir e implementar casos prácticos en los que se pretende que los estudiantes afronten una situación lo más cercana posible a la realidad de manera que, utilizando como base sus conocimientos, realicen una evaluación previa de proyectos basados en aplicaciones energéticas de la biomasa hasta llegar a una propuesta de solución factible que posteriormente tengan que compartir con el resto de compañeros. El objetivo principal asociado a esta actividad es inducir al análisis de una situación de interés y su respuesta sintetizada, es decir, se busca que mediante la reflexión (individual o en grupo) se consiga un grado de análisis que provoque en el alumno una visión de conjunto propia.

\section{Metodología utilizada}

La metodología utilizada se conoce como Estudio o Método del Caso y ha sido estudiada con anterioridad por varios autores (de la Torre, Oliver, Violant, Tejada, Rajadell y Girona, 2003-2004). De acuerdo a Imbernon y Medina (2005), esta metodología se puede implementar mediante dos vías distintas:

a) Cada participante lee el estudio individualmente y contesta brevemente a las preguntas. Posteriormente comienza una discusión general dirigida por el/los profesor/es.

b) También puede dividirse la clase en diversos grupos en los que se discute la solución durante el tiempo que haga falta dependiendo de la complicación y extensión del caso propuesto. Cada grupo nombra un portavoz o portavoces y se realiza la exposición y discusión final. Esta estrategia se recomienda para problemas difíciles y complejos.

Ambas vías son las que utilizan los autores del presente artículo a la hora de implementar las sesiones de casos prácticos, ya que se considera que esta metodología provoca la participación activa, motiva y enseña a analizar problemas implicando al estudiante, por lo que es una estrategia muy eficaz para ponerse en contacto con ideas diferentes, incluso contrarias a las propias. Además, según Román (2003-2004), el método del caso también puede entenderse como un concepto constructivista de la enseñanza y el aprendizaje ya que la reflexión en grupo sobre un caso práctico sirve, tanto al alumno como al profesorado, en el aspecto de formación psicopedagógica.

\section{Objetivos paralelos de la práctica}

Los objetivos relacionados con el aprendizaje son:

- Desarrollar en el alumnado destrezas en la resolución de problemas concretos en un breve período de tiempo.

- Trabajar en grupo de una forma colaborativa y participativa para provocar el intercambio y el debate de opiniones hasta encontrar una posición común. 
- Relacionar contenidos teóricos de diferentes materias de forma práctica.

- Desarrollar en el alumnado un espíritu solidario.

- Desarrollar las capacidades de oratoria y de síntesis.

En cuanto a los objetivos sectoriales referentes a las energías renovables, se pretende:

- Dar a conocer la problemática energética global y sus relaciones con la actividad humana, desarrollando la capacidad de análisis de conflictos socio-ambientales.

- Promover el papel que deben tener las energías alternativas en la sociedad actual, en particular, de la biomasa.

- Utilizar los conocimientos adquiridos previamente hacia la búsqueda de soluciones a los problemas medioambientales.

- Incluir aspectos científicos, tecnológicos, económicos, sociales y medioambientales en la resolución de problemáticas técnicas reales.

Los casos prácticos pretenden fomentar el aprendizaje activo ("aprender haciendo"), parten de la reflexión, promueven el debate y la planificación grupal, y además, requieren de cálculos y de la entrega de una propuesta final. De esta manera, todos los procesos se complementan entre sí, ofreciendo una formación global que contribuye a desarrollar habilidades del pensamiento, competencias y talentos a la vez que se logran afianzar los conocimientos técnicos en lugar de limitar las clases a la tradicional transferencia de información unilateral que produce una actitud pasiva en el alumnado.

\section{Implementación práctica de la metodología}

En primer lugar, es necesario distribuir a los alumnos en grupos, normalmente de entre 5 y 6 personas. Este número permite la existencia de debate interno en el propio grupo, el reparto de tareas entre ellos y la presencia de un ambiente multidisciplinar. Según los contenidos de la práctica, los profesores forman los grupos de manera aleatoria o bien según el perfil y experiencia para configurar grupos adecuados a la tarea. De esta manera se busca que, durante los distintos casos prácticos de cada Módulo de la asignatura, cada alumno tenga diferentes compañeros de grupo.

En cuanto a la organización del tiempo, los casos prácticos se desarrollan conforme a la siguiente estructura y duración aproximada común:

- Introducción teórica (opcional). Duración $\approx 30$ minutos.

- Presentación del caso y de los objetivos del trabajo. Duración $\approx 10$ minutos.

- Lectura de materiales y ejecución del trabajo. Duración $\approx 2,5$ horas.

- Exposición pública y debate. Duración $\approx 30$ minutos.

- Intervención de los profesores haciendo síntesis de las soluciones, aportando sus correcciones y sus propuestas. Duración $\approx 20$ minutos.

Esta distribución es flexible, puesto que la vía de aplicación de la metodología y la estructura y la organización de las sesiones pueden variar de un módulo a otro.

Para los casos correspondientes a los Módulos 2, 4 y 5, basados en la vía grupal, el aula se organiza de manera que haya tantos núcleos de mesas como grupos para 
la resolución del caso (generalmente, 6 núcleos o grupos). Se ubica a los alumnos en el grupo correspondiente buscando cumplir el ambiente multidisciplinar, llevándose a cabo la presentación del caso o situación de interés. Después de la clase de introducción teórica (Módulo 2) y la exposición detallada del caso, el profesor o profesores van pasando por cada grupo durante el período de realización del trabajo, actuando principalmente como gestores en la resolución de dudas y en la aclaración de objetivos y/o de actividades a desarrollar, así como redirigiendo el grupo si se sale del objetivo de la práctica. De esta manera, al finalizar el ejercicio se consigue afianzar los conceptos teóricos que cada profesor considera indispensables para la formación del alumno, es decir, el desarrollo de la práctica por parte de cada grupo y su interacción con el profesorado durante la realización de la misma. Esto a su vez, puede considerarse como una expansión en las acciones tutoriales, las cuales promueven un importante empuje al cambio docente requerido por el nuevo Espacio Europeo de Educación Superior (Gairín, Feixas, Guillamón y Quinquer, 2004).

Una vez acabado el tiempo de resolución del caso, cada grupo entrega su trabajo en forma de presentación visual (PowerPoint o similar) y en ese momento entra en escena un tribunal formado por integrantes del grupo BERA para dar comienzo a la sesión de exposición oral de los trabajos y su consiguiente evaluación.

Es importante que todos los grupos hayan acabado la resolución de su propio caso y escuchen con atención las presentaciones del resto. Así, afianzan a través de las opiniones de sus compañeros los conceptos claves y observan cómo otros han desempeñado el trabajo grupal, cómo se han organizado, etc., de tal modo que vayan mejorando sus competencias y aptitudes para los siguientes casos prácticos y, de manera general, para su futura vida laboral.

En el caso práctico del Módulo 3 (Impactos globales de las políticas de biocarburantes) basado en la vía de aplicación individual, el alumno lee los materiales y aporta sus impresiones, conclusiones y críticas en un foro de debate. Por tanto, durante el período de lectura y ejecución, no hay un tutorado activo por parte de los profesores. El trabajo individual no requiere más de 30 minutos de lectura, aunque se fomenta que el alumno amplíe su visión con materiales optativos y mediante la búsqueda en Internet. En este caso, el peso de la capacidad de argumentación y seguimiento activo del debate cobran especial relevancia, mientras que en los casos basados en la vía grupal prima la adecuada justificación de las decisiones tomadas de forma consensuada dentro del grupo a lo largo del desarrollo práctico, así como la correcta exposición de las mismas ante el tribunal de evaluación.

\section{Recursos empleados para la realización del trabajo}

Con el fin de reproducir lo mejor posible la situación que se podría encontrar en un grupo de trabajo de proyectos a nivel empresarial o de un centro tecnológico, se consideran esenciales los siguientes aspectos:

- La presentación por parte de los profesores debe ser precisa, minuciosa, y ha de definir perfectamente la problemática a solucionar, como lo haría un jefe de proyectos.

- El material aportado debe ser suficiente y no desmedido ni enrevesado. 
- El lugar de trabajo debe permitir una organización espacial cómoda: mesas móviles, espacio suficiente, etc.

- Se han de suministrar ordenadores portátiles (1 ó 2 por grupo) para la implementación de cálculos y de la presentación pública.

- Se ha de proveer de acceso a Internet, con el fin de fomentar la creatividad y búsqueda de soluciones de casos reales parecidos que puedan consultarse en la red.

\section{Métodos de evaluación}

Los evaluadores valorarán los siguientes puntos:

- Comprensión del problema y búsqueda de una solución razonable: 25\%.

- Actitud del grupo para afrontar un caso, participación de todos los alumnos en el trabajo grupal y coordinación para el reparto de este trabajo: 50\%.

- Calidad de la argumentación y la exposición pública concisa: 25\%.

Todos los alumnos de un mismo grupo reciben la misma nota. Finalmente, cada caso práctico supone un $10 \%$ de la nota final del alumno, por lo que la contribución de dicha actividad al resultado total en la asignatura se considera significativa.

\section{DeSARROLlO DE LAS EXPERIENCIAS}

Los casos prácticos implementados en la asignatura "Energía de la Biomasa" son:

- Módulo 2:

- Generación de calor con biomasa sólida.

- Generación de electricidad con biomasa sólida.

- Módulo 3: Impactos globales de las políticas relativas a la promoción y al uso de los biocarburantes.

- Módulo 4: Tratamientos de biomasa residual húmeda.

- Módulo 5: Tratamiento integral de los residuos sólidos urbanos (R.S.U.).

\section{Primer caso práctico del Módulo 2: Generación de calor con biomasa}

Durante este primer caso práctico se persigue que los alumnos utilicen los conceptos enseñados desde el comienzo de la asignatura para desarrollar un caso real de utilización energética de biocombustibles sólidos. Se utilizan nociones estudiadas en las clases de fuentes y caracterización de biocombustibles, de pre-tratamientos, de transporte y almacenamiento $y$, por último, de combustión y efectos asociados a las cenizas, de manera que se combinen todos estos conceptos para la resolución de un caso concreto.

La realización práctica comienza con la organización de los alumnos en grupos. A continuación, el profesorado presenta brevemente los distintos sistemas que configuran una instalación de generación de calor con biomasa, expone los datos técnicos y económicos necesarios para dimensionarlos y, finalmente, explica la metodología según la cual deben realizar el caso práctico, cómo deben desarrollar- 
lo, cómo deben presentar su respuesta al caso y cómo se les evaluará. Posteriormente, se reparten los enunciados de los problemas que tienen que resolver y exponer durante la sesión. En total, se tienen tres enunciados distintos, es decir, que para cada caso habrá dos grupos resolviéndolos y proponiendo una solución particular. Estos se corresponden con las tres escalas de generación de calor con biocombustibles sólidos: nivel doméstico (para uso en casa individual o pequeño edificio), nivel de barrio (en redes de calefacción distribuida) y a gran escala (en industrias).

Al comienzo de la práctica en sí, se entregan los siguientes materiales a los alumnos y con ellos empiezan a trabajar:

- Un recordatorio general sobre la sesión, igual para todos los alumnos. En este se describe el objetivo principal, las actividades a desarrollar o preguntas a las cuales contestar, así como el método de evaluación utilizado.

- Un guión para cada caso práctico, de una página y distribuido a los alumnos del grupo correspondiente, con los datos de partida para la resolución del caso.

- Una lista de empresas, fabricantes, distribuidores, etc., con sus webs, donde se puede encontrar información técnica como apoyo a lo largo del desarrollo.

En cuanto a la resolución técnica, cada grupo debe solventar los principales problemas que puedan existir en el dimensionado de una instalación de generación de calor con biomasa sólida. En primer lugar, se debe seleccionar entre un amplio abanico de posibilidades el combustible y el modelo de caldera adecuados. Para ello, la comparación de las características técnicas y económicas de cada combustible y tecnología de combustión es de vital importancia. En segundo lugar, los alumnos deben calcular el volumen del silo de almacenamiento del combustible y elegir el sistema de transporte entre el silo y la caldera, dependiendo de las limitaciones facilitadas por el/los profesores, las cuales varían de un grupo a otro. Por último, los grupos han de evaluar los riesgos en cuanto a seguridad contra incendios en toda la instalación y seleccionar los sistemas auxiliares para prevenirlos y para adaptarse a la legislación vigente en este ámbito.

Una vez completados los cálculos técnicos y las presentaciones, se procede a la exposición pública tal y como se ha detallado anteriormente.

\section{Segundo caso práctico del Módulo 2: Generación de electricidad con biomasa}

El objetivo principal de este caso práctico es revisar los sistemas de generación eléctrica y cogeneración con biocombustibles sólidos, centrándose en aquellos que representan tecnologías de mayor penetración en el mercado y que se encuentran en un estado de desarrollo, al menos, pre-comercial. Por tanto, debido a que no existe una sesión previa que aborde directamente esta temática, en este caso es necesaria una introducción teórica descriptiva acerca de las diferentes tecnologías y sus principales características técnicas, así como de los principales problemas asociados a su uso con biocombustibles sólidos. Una vez realizada dicha explicación teórica $(\approx 30$ minutos) se procede al planteamiento y resolución del caso práctico, en el que se pone a los diferentes grupos en el papel de empresas que compiten por la concesión de un sistema de cogeneración para un edificio municipal de nueva construcción. 
Se dividen los alumnos en cinco grupos con diferentes roles (similar a lo que se hace en la metodología de "juego de roles" descrita por Imbernon y Medina, 2005):

- Cuatro grupos (dos y dos) representan empresas especializadas en la comercialización de las dos tecnologías de mayor penetración en el mercado (Gasificación + Motor Alternativo de Combustión Interna y Combustión + Ciclo Orgánico de Rankine) y cuyos resultados del análisis tecno-económico son similares, de acuerdo a los datos iniciales facilitados por los profesores. Por tanto, estos cuatro grupos deben defender estas tecnologías exponiendo sus ventajas y planteando soluciones que minimizarán sus limitaciones de cara a que el consejo municipal (tribunal) decida la mejor opción.

- El grupo restante tiene el papel de consultoría externa contratada por el ayuntamiento para dar su opinión acerca de los sistemas que ofertan las empresas interesadas en la concesión. Por tanto, este grupo debe destacar las ventajas y limitaciones de cada una de las tecnologías para poder dar su opinión argumentada acerca de la más adecuada.

Una vez presentado el contexto del caso práctico, los profesores sugieren una secuencia de actividades para la resolución práctica, de manera que cada grupo pueda obtener los resultados de un análisis tecno-económico preliminar que sea la base de sus presentaciones frente al tribunal. Sin embargo, se insta a los alumnos a utilizar los resultados de este análisis como complemento, ya que deben poner especial atención en aspectos que podían decantar la elección del ayuntamiento a su favor.

Tras realizar la secuencia de actividades propuesta, cada grupo está en posición de trabajar en la exposición pública para defender el rol que le ha sido otorgado. Esta es la parte más importante de la realización del caso práctico ya que la valoración de la presentación pública depende de que se justifique debidamente por qué la solución propuesta es la mejor. Como el enunciado es el mismo para todos los grupos pero la solución varía dependiendo de qué grupo lo resuelva, se espera que los resultados finales difieran entre sí, y de esta manera se pueda generar un debate final que enriquezca el criterio de cada alumno, basándose en criterios económicos, energéticos o tecnológicos entre otros.

\section{Caso práctico del Módulo 3: Impactos globales de las políticas de biocarburantes}

Este caso se diferencia substancialmente del resto de casos, tanto por la metodología como por las herramientas utilizadas. En primer lugar se utiliza la plataforma de herramientas web del Anillo Digital Docente (ADD) de la Universidad de Zaragoza. Cada alumno accede con una cuenta personal a un entorno web en el que existen varios foros para la realización de un debate.

Los alumnos descargan de la aplicación web los materiales obligatorios a leer (2 hojas), en el que se describen:

- La importancia de los biocarburantes líquidos para el desarrollo de transporte sostenible y los objetivos marcados.

- Los posibles impactos en el sector agroalimentario y aumento de precios agrícolas. 
- Los cuatro casos particulares en que las políticas de biocarburantes están creando impactos locales graves en cuanto a derechos civiles, de educación y de sanidad:

- Caso de deforestación en Borneo con plantación de palma de aceite.

- Caso de expropiación de tierras agrícolas de campesinos en Colombia y paramilitarismo para plantar palma de aceite.

- Caso de desplazamiento campesino en Argentina para plantaciones de soja.

- Caso de desplazamiento de campesinos, apropiación de recursos hídricos y tierras en Nigeria por compañías transnacionales.

Con la lectura de este material, al alumno se le está planteando si las ventajas socioeconómicas y ambientales de los biocombustibles líquidos, que se estudian en el módulo, lo son tanto dentro como fuera de las fronteras europeas. En el fondo, se espera haber creado un pequeño impacto que induzca a la reflexión, y a la necesidad de leer el material complementario o realizar una búsqueda en Internet. Es por ello que el debate se lleva a cabo a lo largo de las dos semanas que dura el módulo de biocarburantes. Los profesores moderan los foros incluyendo nuevos matices y replanteando las preguntas originales, que eran más bien generalistas, a las más concretas. Cuando la asignatura toca a su fin, los profesores hacen un balance de las opiniones aportadas en los tres foros, configurando un documento resumen.

\section{Caso práctico del Módulo 4: Tratamientos de biomasa residual húmeda (BRH)}

El objetivo principal de este caso práctico es dar una amplia visión de los modos de aprovechamiento y tratamiento de la $\mathrm{BRH}$, que debido a su procedencia (purines de ganado, aguas residuales urbanas o aguas de industrias alimentarias, entre otros) deben ser gestionados de manera adecuada. A través de su resolución se busca que el alumno pueda observar todos los factores económicos, ambientales y sociales a tener en cuenta al proponer una solución práctica para la gestión de la BRH.

En primer lugar se explica el caso práctico en 10 minutos: cada grupo debe aportar una solución para la gestión de los purines de un término municipal de la provincia de Huesca, saturado de granjas porcinas, y en el que existen vertidos incontrolados en campos, acequias y ríos.

Una vez explicado, se reparten a cada grupo sus materiales de trabajo. Los alumnos que participan en cada equipo son elegidos por el profesorado previamente al comienzo de la clase, buscando introducir perfiles adecuados en los grupos 1 a 5, y un perfil lo más multidisciplinar posible en el grupo 6, ya que cada grupo representa una empresa especializada en una disciplina concreta, que deberá presentar una solución viable y convincente para la municipalidad y los vecinos por su eficacia, factibilidad y bondad ambiental:

- Empresa 1: empresa especializada en la producción de biogás por la biometanización de purines. Propone una planta centralizada para digerir los purines de todas las granjas.

- Empresa 2: especializada en instalaciones pequeñas y medianas de biometanización, y que propone hacer una instalación anexa a cada granja, evitando así la creación de una red de transporte de purines. 
- Empresa 3: especializada en secado de materiales, propone utilizar gas natural para generar energía eléctrica, y con el calor residual secar el purín.

- Empresa 4: propone producir compost a través del purín (previamente espesado) y de residuos agrícolas existentes en la municipalidad (paja, restos de podas) y comerzializarlo.

- Empresa 5: propone utilizar los purines directamente como abono nitrogenado, mostrando su factibilidad, las dosis y los ahorros para el sector agrícola local.

- Empresa 6 (opcional): se trata de una ingeniería ambiental. Esta empresa conoce los objetivos del resto de empresas, y debe poner en tela de juicio si las razones aportadas por cada empresa son verdaderas. En el fondo, juega el papel de asesor municipal.

Los grupos deben convencer a la municipalidad de que su solución es la mejor frente al resto de alternativas existentes, excepto para el grupo de la Empresa 6 que es una consultora independiente. A cada grupo se le facilita:

- La descripción del caso y de la empresa, así como objetivos y tareas a realizar.

- Documentos técnicos sencillos de los que los alumnos pueden obtener los datos para hacer los dimensionados sencillos y reforzar la argumentación de las ventajas sociales y ambientales de su instalación.

- La presentación base de su empresa en formato PowerPoint y hoja de cálculo predefinida para facilitar que los alumnos realicen sus cálculos.

El trabajo grupal consiste, en primer lugar, en realizar cálculos de predimensionado y análisis tecno-económico de instalaciones que deben ser capaces de dar tratamiento al total de purines producidos en las granjas. Además deben preparar para la presentación una serie de ventajas de su instalación, y mostrar los problemas ambientales que permite resolver. Para ello, se proporciona un tiempo de 2,5 horas.

La exposición de los grupos se realiza a posteriori, en la que se busca una presentación clara, efectiva y convincente de 5 minutos, ya que el objetivo es vender la solución como óptima para la municipalidad, representada por el tribunal que hace especial hincapié en los factores ambientales y la conveniencia de cara a los ojos de los habitantes (resaltando los posibles rechazos sociales).

\section{Caso práctico del Módulo 5: Tratamiento integral de R.S.U.}

El objetivo principal de este caso práctico es dar a conocer y hacer entender al alumno los aspectos económicos, tecnológicos y medioambientales de mayor relevancia asociados a la gestión de los residuos sólidos urbanos (R.S.U.). En primer lugar, se realiza una introducción teórica estructurada en 5 partes impartidas en una hora: definiciones básicas, legislación, generación de residuos, gestión y tratamiento e impacto ambiental. Se enfatiza en la jerarquía de la gestión integrada de los $\mathrm{RSU}$, que por orden de preferencia es: prevenir, minimizar, reutilizar, reciclar, transformar en energía y verter o disponer.

Durante la exposición oral de los contenidos se propicia a su vez el debate entre los alumnos para afianzar, mediante su propia experiencia, el proceso de gestión de los residuos. 
Una vez finaliza la exposición oral del profesor se da inicio al caso práctico propiamente dicho, en el que los docentes organizan a los estudiantes en grupos. El caso práctico consiste en la aplicación de los conocimientos adquiridos en la exposición oral previa que, además, han sido afianzados mediante el debate, para ofrecer la mejor alternativa de gestión de R.S.U. en un caso real (o similar) seleccionado bien dentro del entorno nacional o del internacional. Cada grupo se considera una empresa de gestión de residuos que ofrece una alternativa de gestión a la comunidad representada por el total de alumnos. De esta manera, los alumnos son quienes además de ofrecer alternativas viables, seleccionan la opción más adecuada entre las opciones proporcionadas por cada empresa. Así, además de impartir nuevos conceptos, el objetivo adicional de la dinámica es afianzar áreas del conocimiento y desarrollar habilidades y destrezas en los alumnos como, por ejemplo, la iniciativa, la creatividad y la toma de decisiones.

Los alumnos disponen de un tiempo de 30 minutos para la resolución del caso, tiempo en el cual el profesor actúa de asesor para la aclaración de las dudas en cada grupo. Finalizado el tiempo, cada grupo (empresa de gestión) ofrece sus ideas de gestión a la comunidad mediante una exposición oral de 5 minutos. Cada grupo puede elegir entre una presentación mediante transparencias o una tipo póster. Durante la exposición de cada grupo el resto de la clase (comunidad) se encarga de evaluar la propuesta considerando dos criterios: a) la calidad de la presentación, tanto desde el punto de vista de la organización de sus contenidos como de la forma de presentarlos visualmente; y b) la justificación de la estrategia de gestión y la toma de decisiones. Un tercer criterio, corresponde a la evaluación de la participación y trabajo en grupo, el cual es juzgado únicamente por el profesor. La media de las calificaciones otorgadas por la comunidad para cada criterio y empresa se compara con la calificación del profesor para otorgar la calificación definitiva obtenida por cada empresa de gestión. Si no existe una diferencia notoria entre ambas calificaciones, se otorga como puntuación final la asignada por la comunidad. En caso contrario, se analizan las diferencias en un diálogo con el grupo evaluado para dar la calificación definitiva. Así, la propuesta definitiva es seleccionada a partir de un criterio consensuado entre los alumnos y el mismo profesor.

\section{RESULTADOS Y DISCUSIÓN}

Los principales resultados de la valoración por parte de los alumnos del Máster Europeo en Energías Renovables, tanto de la docencia en general como de la metodología de casos prácticos pueden observarse en la Tabla 1. Estos resultados se han obtenido de las encuestas que cumplimentan los alumnos tras la finalización de cada asignatura, las cuales intentan recoger la valoración de los principales aspectos docentes.

Sobre los resultados reflejados en la Tabla 1 se puede extraer que, en general, los alumnos no solo prefieren la metodología de casos prácticos que se presenta en este artículo frente a los trabajos en grupo tradicionales, sino que también existe un alto nivel de satisfacción con la metodología, el grado de aprendizaje alcanzado y su aplicabilidad. Muestran, además, un elevado interés por las actividades programadas y el funcionamiento de los grupos de trabajo. Estos resultados positivos concuerdan con los obtenidos por otros autores que han aplicado metodologías similares en relación 
con la comparación de metodologías participativas frente a métodos tradicionales (Molina, Gil, Bravo y Herrero, 2010); en lo referente a la motivación y rendimiento de los estudiantes (González, Pérez, Martínez, Arranz, Esandi, Hernández y otros, 2006, septiembre); o en lo que concierne al potencial de transferencia de aprendizaje y el trabajo en grupo (Romero, Jara, Marín, Millán y Carrillo, 2009, junio).

Tabla 1. Resumen de resultados medios de valoración del alumnado para los cursos 2008/09, 2009/10 y 2010/11 relativos a la docencia y la metodología de casos prácticos (Porcentajes y valoración relativos a 110 encuestas sobre 178 posibles)

\begin{tabular}{|c|c|c|}
\hline & Aspectos a valorar & Valoración media \\
\hline \multirow{4}{*}{ Docencia } & Valoración general de los docentes & $8,72 / 10$ \\
\cline { 2 - 3 } & Dominio de la materia & $8,84 / 10$ \\
\cline { 2 - 3 } & Relación profesor / alumno & $8,87 / 10$ \\
\cline { 2 - 3 } & ¿Te gustaría tenerlos otra vez como docentes? & Si (99\%); No (1\%) \\
\hline \multirow{3}{*}{ Metodología de } & $\begin{array}{c}\text { Valoración de metodología en comparación } \\
\text { con trabajos en grupo tradicionales }\end{array}$ & $\begin{array}{c}\text { Mejor (79\%); Igual (17\%); } \\
\text { Peor (4\%) }\end{array}$ \\
\cline { 2 - 3 } & Satisfacción con la metodología empleada & $8,13 / 10$ \\
\cline { 2 - 3 } & Aprendizaje alcanzado y aplicabilidad & $7,67 / 10$ \\
\cline { 2 - 3 } & Interés por las actividades programadas & $8,00 / 10$ \\
\cline { 2 - 3 } & Funcionamiento del grupo & $7,83 / 10$ \\
\hline
\end{tabular}

En cuanto a los comentarios que realizan los alumnos en las encuestas, las principales críticas y/o recomendaciones son las siguientes:

- Los estudiantes han observado como un punto negativo que no se entregue previamente material específico base para la realización del caso práctico. Este factor hace que los alumnos, que disponen de un tiempo que ellos estiman muy reducido para la elaboración del trabajo, cuenten con una desventaja adicional: el uso de materiales base que, aunque se fundamentan en los contenidos de las clases, son relativamente nuevos para ellos y, por tanto, todavía no del todo asimilados.

- Otro de los aspectos más resaltados por los alumnos es la falta de tiempo para asimilar el objetivo y elaborar la solución final. Se han valorado dos posibilidades para mejorar este aspecto. En primer lugar, se podría dividir las sesiones en dos partes, de tarde y de mañana, en días consecutivos de manera que haya un espacio de tiempo de maduración de ideas entre ambas. Sin embargo, el aumento de la duración de la sesión, que para el alumnado se percibe como una mejora, no siempre lo es tanto para el profesorado, pues se considera que el aprender a trabajar en una solución viable en un corto espacio de tiempo es una situación normal en la realidad laboral. En segundo lugar, se está valoran- 
do, para posteriores ediciones del Máster y en algunos casos prácticos, la constitución de los grupos de trabajo una semana antes de las sesiones, además de la entrega de material introductorio específico con esa misma antelación, lo que podría ser beneficioso para el aprendizaje de los alumnos.

- Se ha sugerido la participación de grupos más reducidos, sin embargo, este aspecto supondría que los alumnos deberían dividirse en un número mayor de grupos, lo que limita el tiempo que los profesores pueden dedicar a cada grupo y, por tanto, el seguimiento del aprendizaje durante la realización de los casos prácticos.

- Se ha propuesto la posibilidad de aumentar el tiempo de las rondas de preguntas y de la evaluación pública por parte de los profesores del tribunal de las soluciones presentadas por cada grupo, pues se cree que permitiría una mejor asimilación de los errores cometidos en los enfoques y cálculos de algunos grupos. Esta posibilidad se baraja positivamente.

- Por último, algún alumno señala la necesidad de designar un Director de Proyectos para dirigir cada grupo de trabajo, que podría hacerse por parte del profesorado y alternando turnos para que cada vez correspondiese a una persona diferente. No se descarta la posibilidad de incorporar esta sugerencia en posteriores ediciones del Máster, de hecho se está barajando la posibilidad de introducir esta medida en uno de los casos prácticos que van a ser impartidos en breve (si el grupo no ha asignado al "líder" antes de un tiempo determinado se elegirá con la ayuda del profesorado), sin embargo, no se ha estudiado en detalle y es posible que sea inadecuada de cara al aprendizaje cooperativo.

A continuación se presentan las impresiones obtenidas por parte del profesorado y los miembros del tribunal relativas al desarrollo de las sesiones prácticas que siguieron la vía de aplicación grupal del Método del Caso:

- Al comienzo los alumnos suelen elegir realizar una lectura personal durante 5 minutos del caso de estudio. Posteriormente, existe un debate sobre el trabajo a realizar y, en general, los grupos priorizan tareas, haciendo una separación en dos o tres subgrupos.

- La interacción con el profesor o profesores es continua. Conceptos claves presentados previamente en sesiones magistrales tienen que ser brevemente reexplicados en determinadas ocasiones.

- La conexión entre los distintos integrantes del grupo a veces es deficiente, generando descoordinación y pérdida de tiempo.

- Se observa que en muchos casos los alumnos no se sienten seguros de los cálculos o de las soluciones aportadas.

- Las presentaciones son en muchos casos desordenados, y parece que los alumnos dan más peso a exponer los cálculos realizados que a transmitir las ideas con claridad o a justificar la opción planteada, pese a que se haya hecho especial hincapié por parte del profesorado en este aspecto al inicio del ejercicio.

- También es importante resaltar que en la realización de los primeros casos prácticos la respuesta de los alumnos puede no llegar a ser todo lo ágil que cabría esperar y, por lo tanto, los profesores deben actuar en consecuencia a la hora de 
interactuar con los estudiantes y evaluarlos. Sin embargo, a medida que van realizando el resto de casos prácticos y se han entendido mejor los objetivos y el funcionamiento del tipo de ejercicio se aprecia una mejora en su forma de organización y trabajo en grupo para conseguir el fin común establecido, lo que refleja la consecución de los objetivos de aprendizaje planteados por los docentes.

En cuanto a la sesión práctica que siguió la vía de aplicación individual, se recogieron las siguientes impresiones:

- Los alumnos tienden en primera instancia a basar sus opiniones en sus propias ideas y conocimientos previos, de manera que los alumnos que ya tienen una opinión formada hacen las aportaciones más críticas.

- Según se desarrolla el debate los alumnos con menor conocimiento se re-plantean su posicionamiento inicial gracias a los comentarios de compañeros con mayor conocimiento.

- Los alumnos terminan por aportar en el debate vínculos a noticias y/o documentos relacionados con el tema que han encontrado en Internet al intentar satisfacer parte de las dudas creadas a raíz del debate.

- Una parte no despreciable de los alumnos se conforma con hacer una intervención con el fin de cumplir con su obligación, sin apenas aportar nada al debate.

- Los profesores tienen que moderar el foro ya que en muchas ocasiones se termina por llegar a una suma de opiniones conformistas similares, que hacen que el debate quede estancado.

\section{CONCLUSIONES}

Las sesiones prácticas planteadas por los autores persiguen dar importancia a los grupos de trabajo multidisciplinares, poniendo en práctica conocimientos básicos de la economía y la ingeniería, así como la búsqueda del afianzamiento de diferentes áreas de conocimiento, la impartición de nuevos conceptos y el desarrollo de habilidades y destrezas por parte del alumnado.

Los autores, que en su mayoría son profesionales iniciándose en la labor docente, encuentran importante que de cara a la formación que debe otorgar un posgrado de las características del Máster Europeo en Energías Renovables impartido por CIRCE, y de cara a las exigencias del Espacio Europeo de Educación Superior, los alumnos ejerciten las habilidades que su futuro desempeño les va a exigir: planificar, trabajar en grupo, hablar en público o hacer una exposición oral ante compañeros. En general, los alumnos se toman muy en serio este tipo de prácticas y responden en consecuencia ya que exigen una formación de calidad. Se trata de una propuesta que les ha motivado, por lo que los autores valoran muy positivamente este tipo de experiencias en contraposición de los sistemas docentes convencionales.

\section{REFERENCIAS BIBLIOGRÁFICAS}

DE LA TORRE, S., OLIVER, C., VIOLANT, V., TEJADA, J., RAJADELL, N. y GIRONA, M. (2003-2004). El cine como estrategia didáctica innovadora. Metodología de estudio de casos y perfil de estrategias docentes. Contextos Educativos, 6-7, 65-86. 
GAIRÍN, J., FEIXAS, M., GUILLAMÓN, C. y QUINQUER, D. (2004). La tutoría académica en el escenario europeo de Educación superior. Revista Interuniversitaria de Formación del Profesorado, 18 (1), 61-77.

GONZÁlEZ, M. L., PÉREZ, C., MARTíNEZ, A., ARRANZ, G., ESANDI, M., HERNÁNDEZ, C., MARTÍNEZ, B., SIMÓN, A. y VIVARACHO, C. (2006, septiembre). Aplicación de metodologías docentes activas en Ingeniería: Experiencia del Grupo GREIDI. Ponencia presentada al 14 Congreso Universitario de Innovación Educativa en las Enseñanzas Técnicas, Oviedo, España.

IMBERNON, F. y MEDINA, J. L. (2005). Metodología participativa en el aula universitaria. La participación del alumnado. Barcelona: Mirador Ediciones.

MOLINA, I. T., GIL, E., BRAVO, I. y HERRERO, R. (2010). Aplicación de la metodología de aprendizaje cooperativo a la materia de Biofarmacia y Farmacocinética. ARS Pharmaceutica, 51 (2), 261-268.

ROMÁN, J. M. (2003-2004). Modelo "CARI" de tutoría de alumnos en la Universidad: Procedimiento de formación de profesores mediante "reflexión en grupo sobre la práctica". Contextos Educativos, 6-7, 43-64.

ROMERO, A., JARA, P., MARÍN, F., MILLÁN, A. y CARRILLO, E. (2009, junio). El Aprendizaje Basado en Problemas (ABP) como práctica multiasignatura con grupos grandes. Experiencia de una práctica ABP en cuatro asignaturas de primer curso de Licenciado en Psicología de la Universidad de Murcia. Ponencia presentada en el Taller Internacional sobre ABP/EBL, Madrid, España.

ROMERO, F., DURÁN, M. M., CARTA, F. y ÁLVAREZ, P. (2002). Metodología docente de aprendizaje desarrollada en la asignatura de Tecnología de los Alimentos. Revista de Enseñanza Universitaria, 20, 149-161.

ZABALZA, M. A. (2003-2004). Innovación en la enseñanza universitaria. Contextos Educativos, 6-7, 113-136.

\section{AGRADECIMIENTOS}

A nuestros estudiantes por su entusiasmo y ganas de aprender. 